\title{
A clash of cosmologies
}

Fundamentalists are threatening astronomy, and astronomy needs to fight back. But this time it's not religious fundamentalists convinced they already know the basic truths of creation. On the contrary, it is precisely because they lack the Universe's basic truths that these 'fundamentalist physicists' have mounted a crusade. They believe that astronomers can provide the truths they want, and they are willing to lay waste the traditions, glories and culture of astronomy to get them. What's worse, some astronomers don't even appreciate the threat.

That, in a nutshell, is the call-to-arms issued by Simon White, director of the Max Planck Institute for Astrophysics in Garching, Germany. In a declaration appearing in this month's Reports on Progress in Physics, and already nailed to the door of the popular arXiv preprint server (http://arxiv.org/abs/0704.2291), White warns his astronomer colleagues that "by uncritically adopting the values of an alien system, astronomers risk undermining the foundations of their own current success." His treatise has been causing a stir in astronomy departments and stoking animated debate on various blogs.

\section{The baryons at the gate}

White argues that astronomers are straying from the true beauty of the field - the study of unusual objects in the sky - into the realm of mere measurement. Particle physicists, a glamorous and well financed bunch, are inveigling astronomers into quantifying fundamental constants to satisfy the equations of cosmology and high-energy physics. White is particularly damning of plans for a mission to study dark energy, a mysterious force that seems to be pushing the Universe apart. Such a project, he says, could suck hundreds of millions of

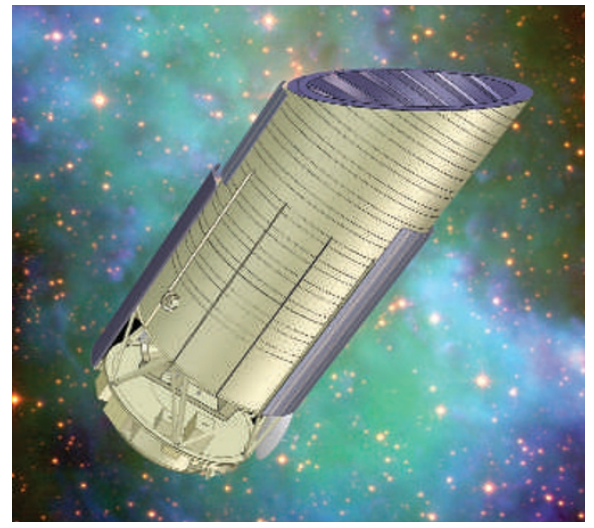

dollars from astronomy in order to measure a single ratio.

"A significant number of astronomers were being browbeaten," White told Nature in an interview. "I wanted to say, 'Hey, there are different ways of thinking about the physical world that are just as interesting as figuring out how particles and forces interact with each other"'

White's paper has quickly found a following among some of his colleagues. "I think it's great," says Paul Schechter, an astronomer at the Massachusetts Institute of Technology. The paper stirred a lively debate at a recent journal club in his department, he says, and many sided with White.

"My first reaction was that there's a lot of truth to this," agrees Charles Steidel, an astrophysicist at the California Institute of Technology in Pasadena. "There's a sort of 'my problem is more fundamental than yours' mentality."

In Europe, support for White's article appears to be even stronger than it is in the United States. The stable, apolitical funding structure in many European countries means that Europeans worry less about the latest fad, says Georg Feulner, an astrophysicist at the Potsdam Institute for Climate Impact Research in Germany. But the United States is so powerful that many Europeans are worried about being dragged along for the ride, says Carlos Frenk, an astrophysicist at Durham University in England. "The US is not Kazakhstan," he says. "It is a driving force in astronomy."

Other astronomers, however, believe that White is both overstating physicists' roles in the field and overlooking their contributions.

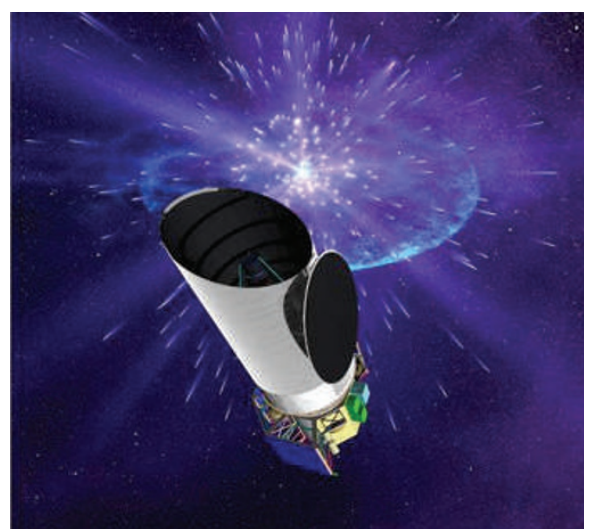

"In the US, dark energy has done more than anything else to reenergize interest in astronomy," says Matt Mountain, director of the Space Telescope Science Institute in Baltimore, Maryland. "I think Simon is just completely wrong."

There's no physicist cabal working against astronomers, adds Roger Blandford, who directs the Kavli Institute for Particle Astrophysics and Cosmology at Stanford University, California. "I don't see particle physics as some sort of dark force out there pursuing dark projects on dark subjects," he says.

No one denies that fundamental physicists have become increasingly involved in astronomy in recent years. The growing entanglement is in part due to the convergence of the two disciplines' theorists on various questions; another factor may be the difficulty that physicists have had in moving beyond their 'standard model' using the traditional tools of their trade - accelerators.

\section{Number crunching}

Physicists have been prominent as designers and builders in projects such as the Wilkinson Microwave Anisotropy Probe (WMAP), which mapped temperature variations of the microwave background, and the Sloan Digital Sky Survey, which catalogued the galaxies across great swathes of the heavens.

The latest project at this junction of astronomy and physics is the one that White portrays as the step too far. America's Joint Dark Energy Mission (JDEM, joint between NASA and the Department of Energy) is currently a

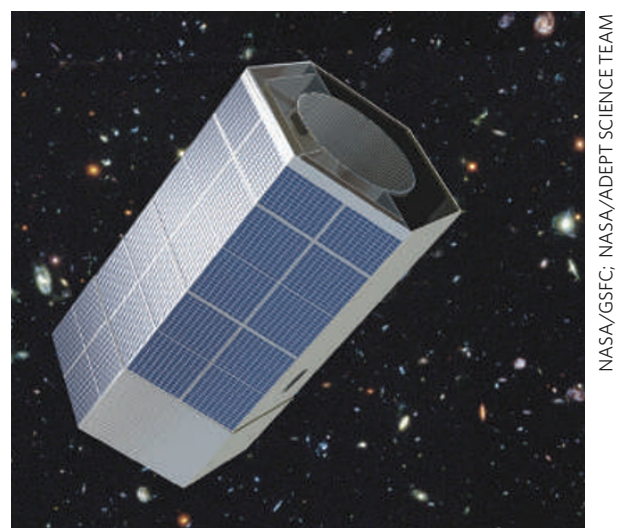

Three possible designs for missions to measure dark energy: SNAP, Destiny and ADEPT. 


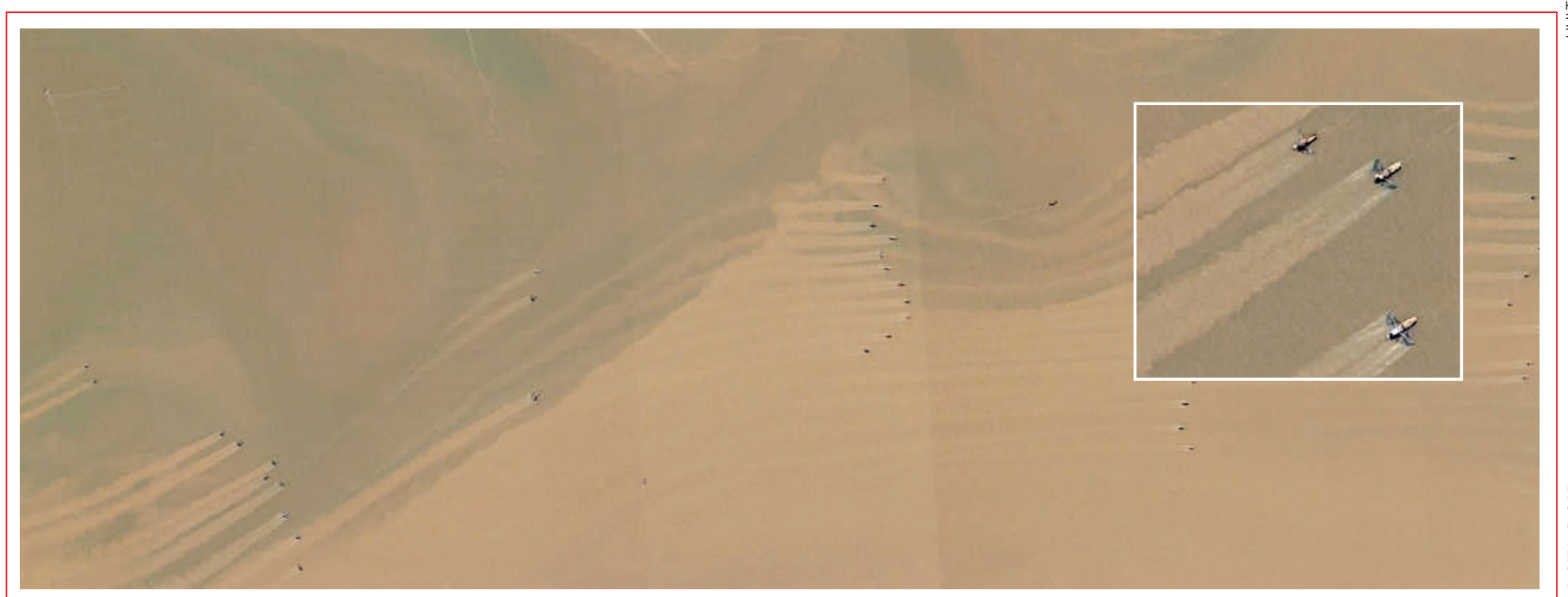

\section{SNAPSHOT \\ Ghosts of destruction}

A set of contrails behind close-flying jets? Orderly raking in a muddy zen garden? A phalanx of harvesters on a giant prairie?

No: this is an image of shrimp trawlers (see inset) off the coast of China. The long plumes of sediment churned up by their nets - 'mudtrails' - are a highly visible sign of the disturbance to sea-bottom ecosystems that they leave in their wake.

Conservation ecologist Kyle Van Houtan of Duke University in North Carolina, and fisheries expert Dan Pauly of the University of British Columbia in Vancouver, have identified many such mudtrails in satellite images available through Google Earth. From the Gulf of Mexico to Malaysia, remote-sensing imagery captures details ranging from the number of trawl nets dragged behind a boat to the white dots of seabirds flocking nearby to feast off the unwanted bycatch that is dumped overboard.

This particular image was taken by the QuickBird satellite on 20 February 2003, off the coast of Jiangsu province near the mouth of the Yangtze River; ten trawlers cover each square kilometre of ocean.

Van Houtan and Pauly are now working with Quickbird, Landsat and other satellite data to quantify exactly how much sediment is churned up by these boats to try to get a handle on the toll taken by fishing. Repeated trawling, they say, can permanently modify the seabed and alter the ecosystem for creatures living in the upper metres of the ocean.

"Imagining is one thing, but imaging is something else," says Van Houtan. "When we see an image, it really crystallizes the impacts and an attitude towards the sea." competition between three different designs for a space telescope that will survey thousands of distant supernovae. But it will do so not out of fascination with the grandeur of exploding suns. Its purpose is simply to gather data on their distances and velocities as a means to improving estimates of dark energy's 'equation of state', a ratio that is a critical parameter in cosmological calculations.

\section{A diet of wormholes}

Astronomers have traditionally built telescopes and satellites that allow them to study a panoply of objects and that enable individuals to pursue their own ideas. In contrast, the new mission would look more like a particle-physics experiment: a large team of researchers would be working exclusively to confirm an already known theory. Such an approach could ostracize the more traditional astrophysicist.
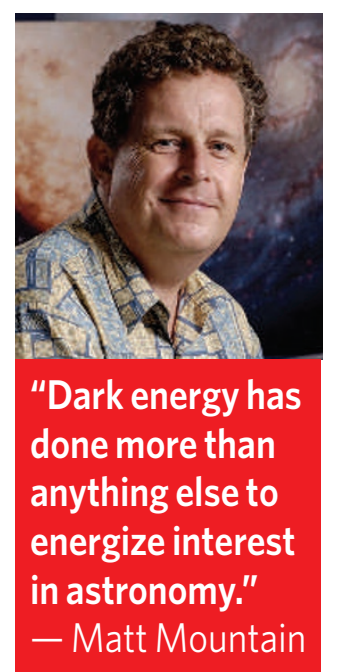

Steidel agrees: confirming theory is only part of what astrophysicists do. "One of the things that make astrophysics interesting to people is that one is almost always surprised," he says. "I think it's that discovery aspect of things that may suffer if resources are directed to answer 'big questions."

Schechter adds that dark energy, although fundamentally interesting, is a murky concept that doesn't have much effect on stars and galaxies - the things most astronomers like to study. "We already know as much about dark energy as we need to know," he says.

Mountain, however, counters with his view that the questions surrounding dark energy are among the most important in the field, and are bringing welcome publicity to astronomy. Furthermore, he says, the missions under consideration for JDEM are a fraction of the cost of the more general-purpose large $\frac{\vec{v}}{3}$ telescopes being advanced by astronomers. And many of the missions would collect data useful to people across the discipline.

Ultimately, Mountain says, White's anxiety is more nostalgia for the good-old days of astronomy than concern for its future. "There's a kind of romantic sense that a lone person with a telescope or a piece of paper should still be able to make breakthroughs in the field." But that's not the way it works in the modern era, he warns. "The contribution of the individual is being lost because some of these problems are getting extraordinarily hard to tackle. The only question is: are we actually losing great science? Or are we just losing the sense that science is as much fun as it once was?"

White stands firm in his belief that the old approach needs to be preserved. "You need to keep the subject vibrant so that it attracts the best young people," he says. "Otherwise the traditional way we do astronomy will cease to function."

Geoff Brumfiel 